An Examination of Inclusivity and Support for Diversity in STEM Fields

\author{
Allison BrckaLorenz ${ }^{\mathrm{a}}$ \\ Heather Haeger ${ }^{\mathrm{b}}$ \\ Christen Priddie ${ }^{\mathrm{a}}$
}

a. Indiana University Bloomington

b. California State University - Monterey Bay

Paper presented at the 2019 Annual Meeting of the American Educational Research Association, Toronto, Canada 


\begin{abstract}
The lack of diversity in STEM professions is an ongoing concern for the US both in terms of social justice and in having a globally competitive workforce. This study provides information for campus leaders to be proactive in considering a wide array of identities to meet the needs of students beyond attending to structural forms of diversity. Data from a large-scale, multi-institution study of students' perceptions of inclusive coursework and institutional commitment to diversity in STEM fields furthers what we know about diversity in these majors. Results encourage campus leaders to additionally consider sexual orientation and disability status in conversations about diversity and to think about how culturally engaging and inclusive courses go beyond the content of the course.
\end{abstract}




\section{An Examination of Inclusivity and Support for Diversity in STEM Fields}

The lack of diversity in science, technology, engineering and math (STEM) professions is an ongoing concern for the United States both in terms of social justice and in having a globally competitive workforce (National Academy of Sciences [NAS], National Academy of Engineering, \& Institute of Medicine, 2011). In order to compete globally, the U.S. will need to increase the overall enrollment, persistence, and representation of people of color in STEM majors (President's Council of Advisors on Science and Technology [PCAST], 2012). Enrollment and persistence of diverse students in STEM majors in college is a critical component in diversifying STEM (NAS, et al, 2011). Greater compositional diversity in college is also beneficial for students and related to greater student recruitment and retention (Astin, 1993; Brown 2006; Cole, 2007; Cole \& Espinoza, 2008; Tinto, 2006).

Previous research has often focused on diversity in STEM in terms of gender, race and ethnicity, and socioeconomic status. This narrow focus ignores other aspects of diversity and inclusion. This study advances that discussion by examining representation in STEM with a broader scope by also looking at inclusion based on sexual orientation and disability status along with examining gender beyond a binary. Additionally, much of the previous research has highlighted the lack of compositional diversity but does not explore the issues of climate or culture in creating diverse environments. Moving beyond looking at diversity in terms of numerical representation and exploring the culture and climate of campuses is also key to understanding diversity in STEM fields (Milem, Chang, \& Lising Antonio, 2005). This research addresses that notion by exploring the roles of institutional commitment to diversity and culturally engaging coursework in diversifying STEM fields.

With the guidance of the Culturally Engagement Campus Environments indicator Proactive Philosophies, this study is intended to lead faculty, administrators, and staff on campus to be proactive in considering a wide array of diverse identities, and creating spaces, support structures, and opportunities that meet the needs of diverse students beyond attending to compositional diversity (Museus, 2014). The indicators in this model represent characteristics of an optimally inclusive and equitable campus environment. One aspect of such an institution would include a support system dedicated to responding to 
DIVERSITY IN STEM

the needs of diverse students. The Proactive Philosophies indicator, more specifically, focuses on an institution-wide philosophy that encourages faculty, staff, and administrators to be proactive in their support efforts. Campus leaders with this philosophy bring information, opportunities, and support to students so that students do not need to put effort toward gathering this information and help for themselves. Hoping for, talking about, and wanting more diverse students to join our STEM programs is not enough. Campus STEM leaders need to be responsible in creating an environment that welcomes and supports all students. To gather critical information for these efforts, we utilize data from the 2017 administration of the National Survey of Student Engagement (NSSE) to address the following research questions:

1. How proportionally represented are students in STEM fields by racial/ethnic identification, first-generation status, gender identity, sexual orientation, and disability status?

2. How does perceptions of institutional commitment to inclusivity and culturally engaging coursework compare for STEM and non-STEM students?

3. Within STEM fields, how do students with different identity characteristics perceive institutional commitment to inclusivity and culturally engaging coursework?

\section{Conceptual Framework}

In understanding diversity and inclusivity in STEM, we approach this topic through a three-tiered lens. First, we look at a more nuanced examination of compositional diversity, then examine inclusivity in coursework within different disciplines, leading to a broader examination of perceptions of the supportiveness of the campus culture. These components of inclusivity are nested and intertwined with compositional diversity or representation at the core of creating a diverse environment. However, compositional diversity is not enough to create an inclusive environment; courses that foster inclusion and intercultural dialog, and a broader campus culture (in and out of the classroom) are necessary to support a diverse student body and create an inclusive environment.

Figure 1. Nested Model for Diversity and Inclusion 


\section{Supportive Campus Environment}

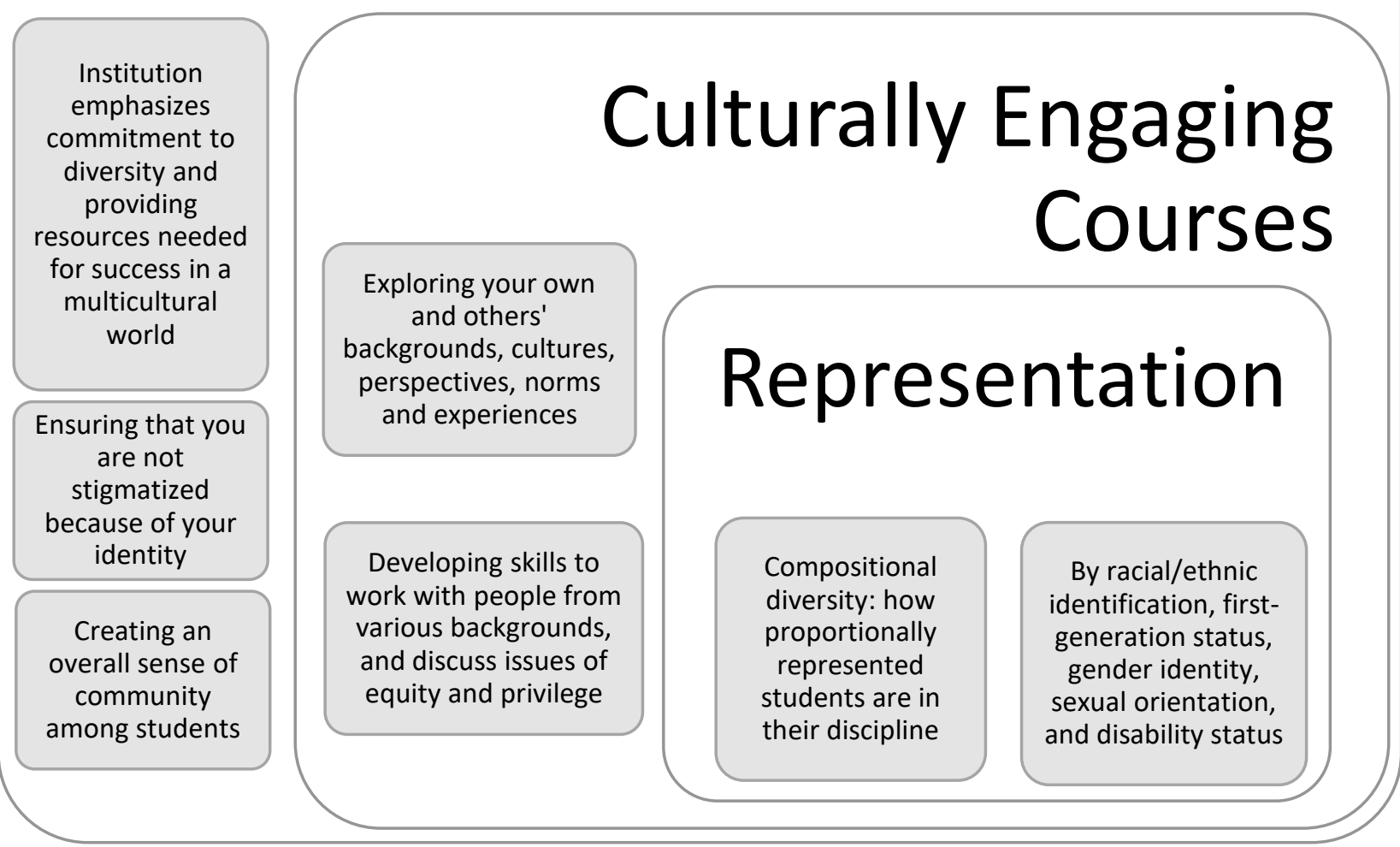

The core of diversity and inclusion is how proportionally represented students are in STEM, and much work is needed in increasing the compositional diversity of STEM majors (President's Council of Advisors on Science and Technology [PCAST], 2012). The disproportional representation can be seen clearly in degrees awarded from U.S. institutions. Though $29 \%$ of the population is from traditionally underrepresented minorities (URMs), only 15\% of STEM Bachelor degrees and $8 \%$ of Doctorates are awarded to URM students (Estrada et al., 2016). A recent study found that when looking at STEM overall, women are just as likely to receive degrees and enroll in graduate school as their male peers; however, specific fields including math, engineering, computer science are still male dominated (Okahana $\&$ Zhou, 2018). This report and much of the research on representation in STEM has focused on race, class, and binary sex (male and female). We expand on this by also looking at how sexual orientation, disability status, and non-binary gender identification are represented in STEM majors. Taking a more 


\section{DIVERSITY IN STEM}

nuanced look at representation is an important foundation not only for creating more equitable access to STEM degrees, but also in the contribution to the higher levels of diversity and inclusion. Studies have also demonstrated that increasing representation is beneficial to the broader campus community in terms of recruitment, retention, and academic success (Astin, 1993; Brown 2006; Cole, 2007; Cole \& Espinoza, 2008; Tinto, 2006). Additionally, Pike and Kuh (2006) demonstrated that as compositional diversity increases, so do diverse interactions among peers and perceptions of more supportive campus environments.

The next level of our model moves from creating a more numerically diverse and representative environment, to examining the degree to which coursework on that campus fosters an inclusive environment. In moving beyond a focus on compositional diversity, we focus on how college coursework can foster a supportive campus environment for a diverse group of students. Culturally relevant pedagogy, which incorporates students' diverse backgrounds and cultures into the classroom, has been associated with a number of positive student outcomes including increased college persistence and graduation (Marquez Kiyama \& Rios-Aguilar, 2017) and retention of content knowledge (Ladson-Billings, 2006). A meta-analysis of research on culturally relevant education (including culturally relevant pedagogy and culturally responsive teaching) found support for culturally relevant education leading to increased engagement and motivation in math and science courses, improved learning in science courses, and advanced understanding of bias and how knowledge is constructed (Aronson \& Laughter, 2016). They also noted that culturally relevant education increased competency in math and science for low-income and first-generation students (Aronson \& Laughter, 2016).

Creating culturally relevant course content is a critical part of supporting students and fostering their development; however, we are also interested in how students engage with course content and with others. Previous studies on culturally relevant education are often focused on specific interventions and then testing the impact of that intervention on a student outcome. We build on this research by focusing instead on students' perceptions of course work and their engagement with culturally relevant pedagogy. We are focusing on culturally engaging courses by measuring how much students engage in discussions 
DIVERSITY IN STEM

with each other and with faculty about their own culture and background, learning about other cultures and perspectives, and engaging in discussions of equity and privilege (see Table 1 for measures of culturally engaging courses). Focusing on how culturally engaging courses are in and out of STEM can help us understand how to students are supported and how they are being prepared to function in a diverse society.

Beyond what happens in the classroom, the overall campus climate and culture is also a critical factor in creating an inclusive campus environment (Milem, Chang, \& Lising Antonio, 2005). Interactions in and out of the classroom along with institutional policies communicate to students the level of institutional commitment to diversity and inclusion (Pike\& Kuh, 2006). We conceptualize a supportive campus environment as students reporting that their institution emphasizes a commitment to diversity, provides students resources for success in a multicultural world, creates a sense of community, and prevents students from being stigmatized because of their identity (see Table1). The culmination of representation, culturally engaging coursework, and institutional commitment to diversity is a supportive campus environment.

\section{Methods}

\section{Data Source}

The data for this study comes from the 2017 administration of the National Survey of Student Engagement (NSSE). NSSE asks students how often they engage in various effective educational practices, their perceptions of the college environment, and how they spend their time in and out of the classroom. This study focuses on respondents from 133 institutions that selected to administer the Inclusiveness and Engagement with Cultural Diversity (ICD) Topical Module. These items examine environments, processes, and activities that reflect engagement with cultural diversity and greater understanding of societal differences. We combined items from the ICD module to create two scales: Course Emphasis and Environment Emphasis. The Course Emphasis scale asked students how much their coursework emphasized such culturally engaging activities as learning about other cultures and respecting the expression of diverse ideas. The Environment Emphasis scale asks students how much their institution 
emphasizes such things as a commitment to diversity and creating an overall sense of community among students. See Table 1 for component items and scale details. Demographic variables of interest are students' major field, class level, racial/ethnic identification, first-generation status, gender identity, sexual orientation, and disability status.

Table 1. Select Inclusiveness and Engagement with Cultural Diversity Items and Scale Information

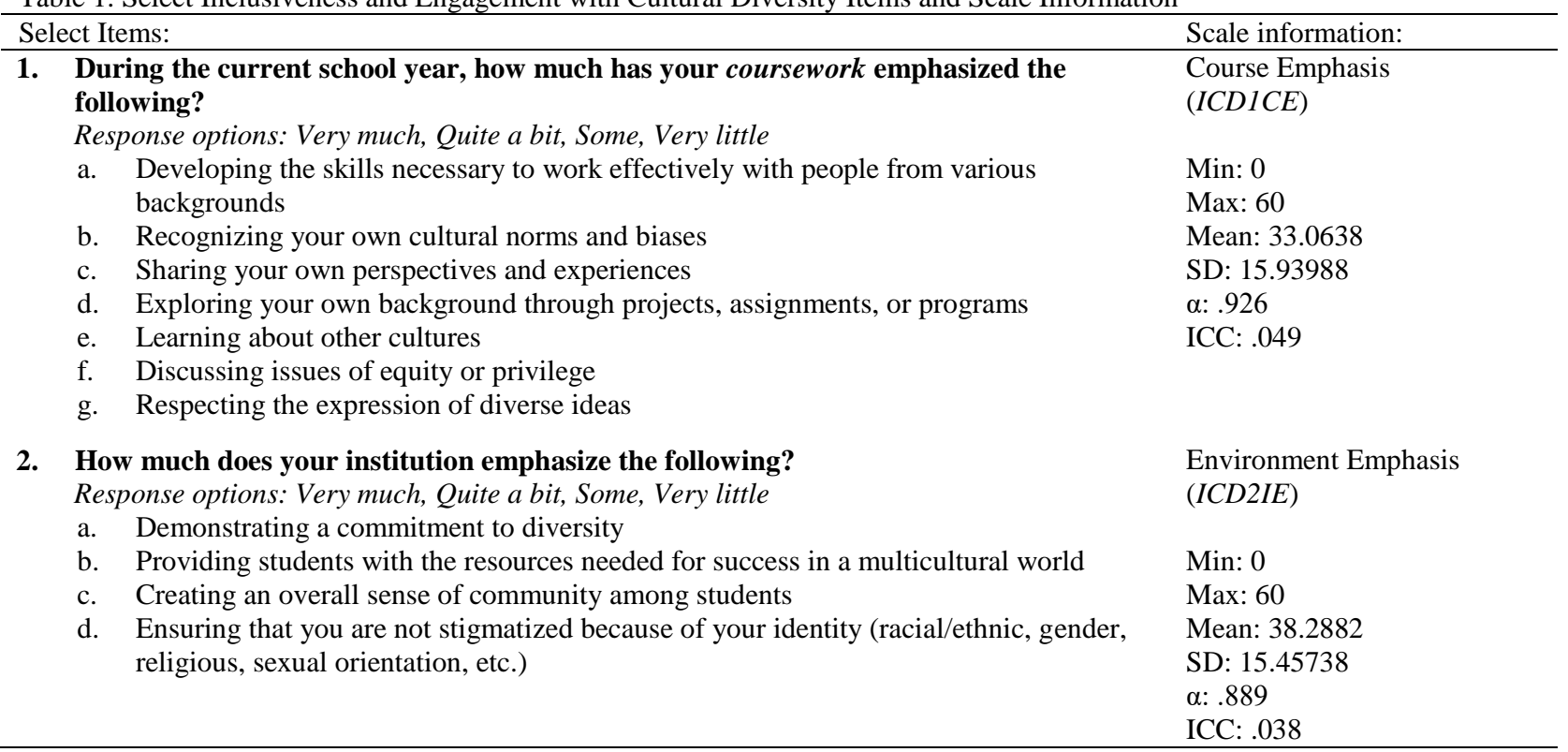

\section{Analyses}

To answer our first research question about how proportionally represented students are in STEM fields by various student demographics, we examined results from chi-square tests. Differences with adjusted residuals greater than 2 or less than -2 were considered notable (Agresti \& Finley, 2009). To answer our second research question about how students' perceptions of institutional commitment to inclusivity and culturally engaging coursework compare for STEM and non-STEM students, we examined a series of regression equations. The dependent variable in models was either the Coursework Emphasis or Environment Emphasis scales. We standardized the dependent variables before entry into models so that unstandardized coefficients can be interpreted as effect sizes. The independent variable of interest was student's major field, collapsed into STEM and non-STEM fields. Table 2 contains information about the coding of additional independent controls. 
DIVERSITY IN STEM

Table 2. Detailed Coding Information for Regression Independent Variables and Controls Major Field

STEM=1 (Students majoring in Biological Sciences, Agriculture, and Natural Resources; Physical Sciences, Mathematics, and Computer Sciences, Engineering fields)

$S T E M=0$ (Students majoring in Arts and Humanities; Social Sciences; Business; Communications, Media, and Public Relations; Education; Health Professions; and Social Service Professions fields)

Racial/Ethnic Identification

Indig $=1$ (American Indian or Alaska Native, Native Hawaiian or other Pacific Islander); Asian = 1 $($ Asian $) ;$ Black $=1$ (Black or African American); Latino $=1$ (Hispanic of Latino); White $=1$ (White); Other $=1$ (Other); Multi = 1 (Selected two or more of the racial/ethnic identities not including "I prefer not to respond); RacePNR = 1 (I prefer not to respond)

Gender Identity Man = $1($ Man $) ;$ Woman $=1($ Woman $) ;$ GenderVar $=1($ Another gender identity $) ;$ GenderPNR $=1(\mathrm{I}$ prefer not to respond)

Disability Status

DisNo $=1$ (Has not be diagnosed with a disability or impairment); DisYes $=1$ (Has been diagnosed with a disability or impairment); $D i s P N R=1$ (I prefer not to respond)

Sexual Orientation

$L G B Q+=1$ (Bisexual, Gay, Lesbian, Queer, Questioning or unsure, Another sexual orientation); Straight $=1($ Straight $($ heterosexual $)) ; \operatorname{SexPNR}=1($ I prefer not to respond $)$

Class level Senior $=1$ (Senior); Senior $=0$ (First-year student $)$

First-generation Status

Firstgen $=1$ (Neither parent or anyone who raised you holds a Bachelor's degree); Firstgen $=0(\mathrm{~A}$ parent or someone who raised you holds a Bachelor's degree)

To answer our final research question about how students with different identity characteristics perceive institutional commitment to inclusivity and culturally engaging coursework within STEM fields, we examined a series of regression models. Models were identical to the models in the second research question except that we limited these models exclusively to students with STEM majors, and we removed the STEM independent variable. We used effect coding to code non-dichotomous multi-categorical demographics so that comparisons could be made to the average STEM student as opposed to a reference group (Mayhew \& Simonoff, 2015). For example, in regression models with traditional dummy coding, a reference group would be left out of the model, say women, resulting in the coefficients of all other gender identity groups being interpreted as being compared to the women reference group. With effect coding, all groups receive coefficients, and these coefficients are interpreted as being compared to the average student in the model, in this case, the average STEM student.

\section{Limitations}

Institutions elect to participate in NSSE and further, they optionally choose to append additional item sets such as the items that are the focus of this study. This may create a selection bias where institutions that choose to administer this module of items are somehow substantively different from other institutions, therefore limiting generalizability. In several situations (American Indian or Alaska Native, 
Native Hawaiian or other Pacific Islander; LGBQ+, and even STEM students) students were aggregated together either to increase group counts or to simplify comparisons. There is great diversity within these and all sub-groups of students studied here so generalizations should be made with caution. Future research could expand on the variation within these sub-populations. Even the grouping of majors into a STEM/non-STEM dichotomy hides variation that likely occurs within individual majors and specific departments. Although we use effect coding to compare sub-groups of students to the average STEM students rather than an arbitrary reference group, this methodology does have its limitations. As we learned in our first research question, there is not a proportional balance among identity characteristics and the "average" STEM student may be more like the majority subgroups in STEM fields. Additionally, subgroups of students may be closer to the "average" student than one another, masking differences between the subgroups themselves.

\section{Results}

\section{Respondents}

The student respondents in this study consisted of 23,068 first-years and 30,436 seniors who responded to the ICD Topical Module item set and reported a major field. To see more details about respondents, see Table 3.

Table 3. Select Student Characteristics by STEM Major

\begin{tabular}{|c|c|c|c|c|}
\hline & & $\begin{array}{r}\text { Non-STEM } \\
\%) \\
\end{array}$ & STEM (\%) & Total $(\%)$ \\
\hline \multirow[t]{2}{*}{ Classification } & First-year & 41.7 & 46.8 & 43.1 \\
\hline & Senior & 58.3 & 53.2 & 56.9 \\
\hline Racial/Ethnic & American Indian or Alaska Native & 0.7 & 0.3 & 0.6 \\
\hline \multirow[t]{8}{*}{ Identification } & Asian & 5.6 & 10.7 & 7.0 \\
\hline & Black or African American & 6.3 & 4.6 & 5.9 \\
\hline & Hispanic or Latino & 7.1 & 5.0 & 6.5 \\
\hline & Native Hawaiian or Other Pacific Islander & 0.2 & 0.2 & 0.2 \\
\hline & White & 68.5 & 66.3 & 67.9 \\
\hline & Other & 1.2 & 1.6 & 1.3 \\
\hline & Multiracial & 7.4 & 7.8 & 7.5 \\
\hline & I prefer not to respond & 3.1 & 3.5 & 3.2 \\
\hline \multirow[t]{2}{*}{ First-Generation Status } & Not first-generation & 56.5 & 66.2 & 59.1 \\
\hline & First-generation & 43.5 & 33.8 & 40.9 \\
\hline \multirow[t]{4}{*}{ Gender identity } & Man & 27.6 & 49.9 & 33.6 \\
\hline & Woman & 70.2 & 47.4 & 64.1 \\
\hline & Another gender identity & 1.1 & 1.4 & 1.1 \\
\hline & Prefer not to respond & 1.1 & 1.3 & 1.2 \\
\hline
\end{tabular}


DIVERSITY IN STEM

\begin{tabular}{|c|c|c|c|c|}
\hline \multirow[t]{8}{*}{ Sexual Orientation } & Straight & 84.9 & 84.6 & 84.8 \\
\hline & Bisexual & 5.1 & 5.1 & 5.1 \\
\hline & Gay & 1.4 & 1.4 & 1.4 \\
\hline & Lesbian & 1.1 & 0.9 & 1.0 \\
\hline & Queer & 1.1 & 0.9 & 1.0 \\
\hline & Questioning or unsure & 1.2 & 1.5 & 1.3 \\
\hline & Another sexual orientation & 1.6 & 1.8 & 1.7 \\
\hline & I prefer not to respond & 3.6 & 3.8 & 3.6 \\
\hline \multirow[t]{3}{*}{ Disability Status } & No & 82.7 & 85.1 & 83.4 \\
\hline & Yes & 13.6 & 11.5 & 13.1 \\
\hline & I prefer not to respond & 3.7 & 3.4 & 3.6 \\
\hline
\end{tabular}

How proportionally represented are students in STEM fields by racial/ethnic identification, firstgeneration status, gender identity, sexual orientation, and disability status?

There was a significant relationship between racial/ethnic identification and major field $\left(\chi^{2}=\right.$ 558.60, $p<.001)$. American Indian or Alaska Native students ( $\mathrm{AR}=-4.7)$, Black or African American students $(A R=-7.6)$, Hispanic or Latino students $(A R=-8.5)$, and White students $(A R=-4.7)$ were underrepresented in STEM fields, while Asian students $(A R=20.3)$ and Other students $(A R=3.6)$ were overrepresented in STEM fields. There was additionally a significant relationship between whether a student was first-generation or not and their major $\left(\chi^{2}=403.78, p<.001\right)$; first-generation students were underrepresented in STEM (AR = -20.1). Gender identity also had a significant relationship with major field $\left(\chi^{2}=2418.93, p<.001\right)$; women were underrepresented in STEM fields (AR = -48.7). Sexual orientation had a significant relationship with major as well $\left(\chi^{2}=19.70, p<.01\right)$. Students who identify as lesbian $(\mathrm{AR}=-2.0)$ and queer $(\mathrm{AR}=-2.5)$ were underrepresented in STEM fields, and students who were questioning or unsure were overrepresented in STEM. Lastly, disability status had a significant relationship with students' major $\left(\chi^{2}=46.93, p<.001\right)$. Students who self-reported having a disability were underrepresented in STEM fields $(\mathrm{AR}=-6.5)$. Table 4 contains additional $\chi^{2}$ details.

Table 4. Chi-Square $\left(\chi^{2}\right)$ Statistics by STEM Field

\begin{tabular}{lrrr} 
& Non-STEM & STEM & \\
\cline { 2 - 3 } Racial/Ethnic Identification & N (Adjusted Residual) & N (Adjusted Residual) & $\chi^{2}(\mathrm{df})$ \\
American Indian or Alaska Native & & & $\mathbf{5 5 8 . 6 0}(\mathbf{8})$ \\
Asian & $261(4.7)$ & $46(-4.7)$ & $<.01$ \\
Black or African American & $2,180(-20.3)$ & $1,525(20.3)$ \\
Hispanic or Latino & $2,466(7.6)$ & $657(-7.6)$ \\
Native Hawaiian or Other Pacific Islander & $2,744(8.5)$ & $714(-8.5)$ \\
White & $70(0.5)$ & $23(-0.5)$ \\
Other & $26,661(4.7)$ & $9,491(-4.7)$ \\
Multiracial & $455(-3.6)$ & $223(3.6)$ \\
& $28,88(-1.7)$ & $1,123(1.7)$
\end{tabular}


I prefer not to respond

First-Generation

Not first-generation

First-generation

Gender Identity

Man

Woman

Another gender identity

Prefer not to respond

Sexual Orientation

Straight

Bisexual

Gay

Lesbian

Queer

Questioning or unsure

Another sexual orientation

I prefer not to respond

Disability Status

No

Yes

I prefer not to respond

$$
1,191(-2.7)
$$

$22,000(-20.1)$

$9,473(20.1)$

$10,782(-48.4)$

$27,439(48.7)$

$411(-2.9)$

$439(-2.0)$

$33,121(0.8)$

$2,001(0.0)$

$544(-0.2)$

$412(2.0)$

$433(2.5)$

$482(-2.1)$

$628(-1.9)$

$1,399(-1.3)$

$32,253(-6.7)$

$5,350(6.5)$

$1,426(1.6)$
$504(2.7)$

$16,928(20.1)$

$4,843(-20.1)$

$7,169(48.4)$

$6,815(-48.7)$

194 (2.9)

$192(2.0)$

$12,145(-0.8)$

$735(0.0)$

$205(0.2)$

$123(-2.0)$

$124(-2.5)$

$210(2.1)$

265 (1.9)

549 (1.3)

$12,210(6.7)$

$1,659(-6.5)$

$482(-1.6)$
$2418.93(3) \quad<.01$

$19.70(7) \quad<.01$

$403.78(1) \quad<.01$

$46.93(2)$

$<.01$

How does perceptions of institutional commitment to inclusivity and culturally engaging coursework compare for STEM and non-STEM students?

STEM students' coursework emphasized significantly and notably less culturally engaging content compared to non-STEM students $(\mathrm{B}=-.514, p<.001)$. The impact of this difference would be large by NSSE standards (Rocconi \& Gonyea, 2018). Additionally, STEM students perceive less of an institutional emphasis on a commitment to diversity $(\mathrm{B}=-.097, p<.001)$ although this difference would be small (see Table 5).

Table 5. OLS Regression Coefficients for ICD Scales

\begin{tabular}{lcccccccc}
\hline & \multicolumn{4}{c}{ Coursework Emphasis } & \multicolumn{3}{c}{ Institution Emphasis } \\
\cline { 2 - 10 } & B & S.E. & $\beta$ & Sig. & B & \multicolumn{2}{c}{ S.E. } & $\beta$ \\
\hline STEM major & -.514 & .010 & -.228 & $* * *$ & -.097 & .010 & -.043 & $* * *$ \\
\hline
\end{tabular}

Note: $* * * p<.001$. Controls include racial/ethnic identity, gender identity, disability status, sexual orientation, class level, and first-generation status.

Within STEM fields, how do students with different identity characteristics perceive institutional commitment to inclusivity and culturally engaging coursework?

Coursework emphasis for STEM students varies by all the demographics examined. Asian $(\mathrm{B}=$ $.159, p<.001)$ and Black or African American $(\mathrm{B}=.175, p<.001)$ students perceive a stronger emphasis 
on cultural engagement as well as women $(\mathrm{B}=.161, p<.001)$, students without a diagnosed disability or impairment $(\mathrm{B}=.076, p<.001)$, straight students $(\mathrm{B}=.061, p<.01)$, and first-generation students $(\mathrm{B}=$ $.064, p<.001)$ compared to the average STEM student. White $(\mathrm{B}=-.200, p<.001)$ and multiracial students $(\mathrm{B}=-.109, p<.001)$ as well as seniors $(\mathrm{B}=-.239, p<.001)$ perceive a less strong emphasis on cultural engagement than the average STEM student. Table 6 contains the full regression results.

Table 6. OLS Regression Coefficients for ICD Scales for STEM students

\begin{tabular}{|c|c|c|c|c|c|c|c|c|c|}
\hline & & \multicolumn{4}{|c|}{ Coursework Emphasis } & \multicolumn{4}{|c|}{ Institution Emphasis } \\
\hline & & B & $\mathrm{SE}$ & $\bar{\beta}$ & Sig. & B & $\mathrm{SE}$ & $\beta$ & Sig. \\
\hline \multirow{9}{*}{$\begin{array}{l}\text { Racial/Ethnic } \\
\text { identification }\end{array}$} & (Constant) & -.367 & .036 & & $* * *$ & -.207 & .036 & & $* * *$ \\
\hline & Indigenous & .102 & .105 & .020 & & .109 & .105 & .022 & \\
\hline & Asian & .159 & .030 & .058 & $* * *$ & .009 & .030 & .003 & \\
\hline & Black or African Am. & .175 & .039 & .049 & $* * *$ & -.007 & .039 & -.002 & \\
\hline & Hispanic or Latino & .023 & .038 & .007 & & .065 & .038 & .019 & \\
\hline & White & -.200 & .022 & -.110 & $* * *$ & -.001 & .022 & .000 & \\
\hline & Other & .061 & .061 & .014 & & -.061 & .062 & -.014 & \\
\hline & Multiracial & -.109 & .033 & -.036 & $* * *$ & -.018 & .033 & -.006 & \\
\hline & Prefer not to respond & -.210 & .047 & -.041 & $* * *$ & -.097 & .047 & -.019 & $*$ \\
\hline \multirow[t]{4}{*}{ Gender identity } & Man & .007 & .029 & .004 & & .114 & .029 & .060 & $* * *$ \\
\hline & Woman & .161 & .029 & .084 & $* * *$ & .181 & .029 & .095 & $* * *$ \\
\hline & Another gender identity & -.056 & .058 & -.009 & & -.223 & .059 & -.037 & $* * *$ \\
\hline & Prefer not to respond & -.112 & .061 & -.059 & & -.072 & .062 & -.038 & \\
\hline \multirow[t]{3}{*}{ Disability status } & No disability & .076 & .019 & .035 & $* * *$ & .074 & .019 & .034 & $* * *$ \\
\hline & Disability & -.005 & .023 & -.002 & & .006 & .023 & .002 & \\
\hline & Prefer not to respond & -.071 & .032 & -.033 & $*$ & -.080 & .032 & -.037 & * \\
\hline \multirow[t]{3}{*}{ Sexual orientation } & LGBQ+ & .043 & .023 & .016 & & .015 & .023 & .006 & \\
\hline & Straight & .061 & .019 & .029 & $* *$ & .067 & .019 & .032 & $* * *$ \\
\hline & Prefer not to respond & -.104 & .032 & -.050 & $* *$ & -.082 & .033 & -.039 & * \\
\hline Class level & Senior & -.239 & .017 & -.119 & $* * *$ & -.229 & .017 & -.114 & $* * *$ \\
\hline Parent education & First-generation & .064 & .018 & .030 & $* * *$ & -.017 & .018 & -.008 & \\
\hline
\end{tabular}

Perceptions of institution emphasis on a commitment to inclusivity varied by gender identity, disability status, sexual orientation, and class level. Men $(\mathrm{B}=.114, p<.001)$ and women $(\mathrm{B}=.181, p<$ $.001)$, students without a diagnosed disability $(\mathrm{B}=.074, p<.001)$, and straight students $(\mathrm{B}=.067, p<$ .001) all felt their institution more strongly emphasized a commitment to diversity and inclusion than the average STEM student. Gender variant students $(\mathrm{B}=-.223, p<.001)$ and seniors $(\mathrm{B}=-.229, p<.001)$ felt this commitment was emphasized less strongly than the average STEM student.

\section{Discussion and Significance}


DIVERSITY IN STEM

Much of the diversity research in STEM fields focuses on binary gender, race and ethnicity, or socioeconomic status, but these results remind us that there is a larger view of diversity that is often not discussed. By examining non-binary gender, sexual orientation, and disability status in addition to firstgeneration status and racial/ethnic identification, we see that there are many identities that students hold that may not be addressed in conversations about diversity. These results demonstrate that continued work is needed to increase the representation of women, students of color, and first-generation students in STEM, but that we see a lack of diversity in STEM fields, not only with race/ethnicity, gender, and socioeconomic status, but we also see that sexual orientation and disability status may be marginalized in STEM environments. Given this disparity, other forms of diversity should be examined and discussed as well. Campus leaders should proactively discuss the ways that messages about welcome, acceptance, and inclusion can extend beyond the current norm for STEM programs.

One area that might be a good place to start is in more inclusive and culturally engaging coursework. Though culturally engaging classes can have a dramatic impact on the retention of students of color in STEM (Jackson, Galvez, Landa, Buonora, \& Thoman, 2016), STEM students were less likely to experience culturally engaging curriculum or perceive institutional commitment to diversity. In order to address the issue of underrepresentation in STEM and the country's need for a larger and more diverse STEM workforce, institutions of higher education need to increase access to culturally relevant coursework in STEM in addition to ensuring that all students feel safe, supported and valued on campus. STEM faculty may feel that cultural norms and topics of diversity are beyond the scope of their content curriculum, but models such as Nelson Laird's (2014) Diversity Inclusivity Framework offer a variety of ways beyond curricular content where courses can be more inclusive such as having instructors explore and better understand their own views, biases, and values of the learners in their courses. Additionally, a meta-analysis on culturally relevant education by field of study found some of the strongest evidence for its benefit in STEM courses (Aronson \& Laughter, 2016). Faculty development that highlights the effectiveness of creating culturally engaging coursework along with strategies for implementing this type of pedagogy in STEM courses could increase access to culturally engaging courses for STEM students. 
DIVERSITY IN STEM

The results from this study further show us that messages about inclusive coursework and institutional commitment are not received equally by diverse types of students - campus commitment to diversity is not monolithic. The perception of commitment to diversity varied both by discipline, with STEM students perceiving lower commitment to diversity, and by student characteristics. Perception of commitment to diversity varied by gender identity, disability status, sexual orientation, and class level. As institutions work to improve their campus climate and demonstrate their commitment to diversity, they should consider this variability to ensure that interventions are culturally relevant to the full diversity of the student population. It is important that campus leaders proactively work toward making the support for students in STEM universal. 
DIVERSITY IN STEM

\section{References}

Agresti, A. \& Finley, B. (2009). Statistical Methods for the Social Sciences. Prentice Hall, Inc.: Upper Saddle River, NJ.

Aronson, B., \& Laughter, J. (2016). The Theory and Practice of Culturally Relevant Education: A Synthesis of Research across Content Areas. Review of Educational Research, 86(1), 163-206. https://doi.org/10.3102/0034654315582066

Astin, A. W. (1993). Diversity and Multiculturalism on the Campus. Change: The Magazine of Higher Learning, 25(2), 44-49. https://doi.org/10.1080/00091383.1993.9940617

Brown. K. (2006) The Educational Benefits of Diversity: The Unfinished Journey from "Mandate" in Brown to "Choice" in Grutter and Comfort. Leadership and Policy in Schools 5:4, pages 325354.

Cole, D. (2007). Do Interracial Interactions Matter? An Examination of Student-Faculty Contact and Intellectual Self-Concept. The Journal of Higher Education, 78(3), 249-281.

Cole, D., \& Espinoza, A. (2008). Examining the Academic Success of Latino Students in Science Technology Engineering and Mathematics (STEM) Majors. Journal of College Student Development, 49(4), 285-300. https://doi.org/10.1353/csd.0.0018

Estrada, M., Burnett, M., Campbell, A. G., Campbell, P. B., Denetclaw, W. F., Gutiérrez, C. G., ... Zavala, M. (2016). Improving Underrepresented Minority Student Persistence in STEM. CBE Life Sciences Education, 15(3). https://doi.org/10.1187/cbe.16-01-0038

Jackson, M. C., Galvez, G., Landa, I., Buonora, P., \& Thoman, D. B. (2016). Science That Matters: The Importance of a Cultural Connection in Underrepresented Students' Science Pursuit. CBE-Life Sciences Education, 15(3), ar42. https://doi.org/10.1187/cbe.16-01-0067

Ladson-Billings G. (2006). "Yes, but how do we do it?" Practicing culturally relevant pedagogy. In Landsman J. G., Lewis C. W. (Eds.), White Teachers Diverse Classrooms: Creating Inclusive Schools, Building on Students' Diversity, and Providing True Educational Equity (pp. 33-46). Sterling, VA: Stylus. 
DIVERSITY IN STEM

Marquez Kiyama, J., \& Rios-Aguilar, C. (2017). Funds of Knowledge in Higher Education | Honoring Students' Cultural Experiences and Resources as Strengths (1st Edition). New York, NY, USA: Routledge. Retrieved from https://www.taylorfrancis.com/books/e/9781315447315

Mayhew, M. J. \& Simonoff, J. S. (2015). Non-White, no more: Effect coding as an alternative to dummy coding with implications for higher education researchers. Journal of College Student Development, 56(2), 170-175.

Museus, S. D. (2014). The Culturally Engaging Campus Environments (CECE) Model: A new theory of college success among racially diverse student populations. In M. B. Paulsen (Ed.), Higher Education: Handbook of Theory and Research. New York: Springer.

Nelson Laird, T. F. (2014). Reconsidering the inclusion of diversity in the curriculum. Diversity and Democracy, 17(4).

National Academy of Sciences, National Academy of Engineering, \& Institute of Medicine. (2011). Expanding Underrepresented Minority Participation: America's Science and Technology Talent at the Crossroads. Washington, DC: The National Academies Press. https://doi.org/10.17226/12984.

Okahana, H., \& Zhou, E. (2018). Graduate Enrollment and Degrees: 2007 to 2017 (p. 82). Washington, DC: Council of Gradaute Schools. Retrieved from https://cgsnet.org/ckfinder/userfiles/files/CGS_GED17_Report.pdf

Pike, G. R., \& Kuh, G. D. (2006). Relationships among Structural Diversity, Informal Peer Interactions and Perceptions of the Campus Environment. The Review of Higher Education, 29(4), 425-450. https://doi.org/10.1353/rhe.2006.0037

President's Council of Advisors on Science and Technology. Engage to Excel: Producing One Million Additional College Graduates with Degrees in Science, Technology, Engineering and Mathematics. Washington, DC: U.S. Government Office of Science and Technology; 2012. 
Rocconi, L. \& Gonyea, R. (2018). Contextualizing effect sizes in the National Survey of Student Engagement: An empirical analysis. Research and Practice in Assessment, 13, 22-38.

Tinto, V. (2006). Research and Practice of Student Retention: What Next? Journal of College Student Retention: Research, Theory \& Practice, 8(1), 1-19. https://doi.org/10.2190/4YNU-4TMB-22DJ-AN4W 